\title{
Differentiated Thyroid Cancer Patients More Than 60 Years Old Paradoxically Show an Increased Life Expectancy
}

\author{
Tobias Markus Maier ${ }^{1}$, Otmar Schober ${ }^{1}$, Joachim Gerß ${ }^{2}$, Dennis Görlich ${ }^{2}$, Christian Wenning ${ }^{1}$, Michael Schaefers ${ }^{1}$, \\ Burkhard Riemann*1, and Alexis Vrachimis*1 \\ ${ }^{1}$ Department of Nuclear Medicine, University Hospital Münster, Münster, Germany; and ${ }^{2}$ Institute of Biostatistics and Clinical \\ Research, University Hospital Münster, Münster, Germany
}

\begin{abstract}
The aim of this study was to compare the overall survival of a large, single-center cohort of patients who had differentiated thyroid cancer (DTC) with that of a matched general population. Methods: We analyzed 2,428 consecutive patients who had DTC and underwent treatment from 1965 to 2013 at the Department of Nuclear Medicine, University Hospital of Münster, Münster, Germany, according to international standards. Patients were classified on the basis of the current, seventh edition of the American Joint Committee on Cancer/ Union for International Cancer Control classification system. Additionally, a subgroup analysis with regard to age at diagnosis was performed. The overall survival of the patients was compared with the expected survival of the general population on the basis of age and sex, as provided by the Federal Statistical Office of Germany. Results: Compared with the expected survival, the overall survival of patients with stage I disease paradoxically was significantly better $(P<0.001)$. In the subgroup analysis, a significantly lower mortality rate was observed in elderly patients ( $\geq 60$ y old) with stage I disease. On the other hand, patients between 20 and 45 y of age and with distant metastases at diagnosis had a significantly increased standardized mortality rate. In contrast, other patients with stage II disease and more than $45 \mathrm{y}$ old had a normal mortality rate. The mortality rate was significantly increased in all patients with stage IVC disease. Conclusion: Older patients with more limited disease paradoxically had better survival than would be expected on the basis of age and sex, whereas young adults as well as patients more than $45 \mathrm{y}$ old and with distant metastases had increased mortality rates. For all other DTC patients, regardless of age or TNM stage, no significant survival difference was seen.
\end{abstract}

Key Words: differentiated thyroid cancer; overall survival; expected survival

J Nucl Med 2015; 56:190-195

DOI: $10.2967 /$ jnumed.114.150284

\section{$\mathbf{M}$}

alignant tumors of the thyroid are the most common malignant endocrine neoplasms, comprising approximately $1 \%$ of all malignant tumors (1). In recent years, there has been a clear rise in the incidence of malignant thyroid carcinomas (2), especially

Received Oct. 20, 2014; revision accepted Dec. 8, 2014

For correspondence or reprints contact: Alexis Vrachimis, Department of Nuclear Medicine, University Hospital Münster, Albert-Schweitzer-Campus 1, Building A1, 48149 Münster, Germany.

E-mail: vrachal@uni-muenster.de

${ }^{*}$ Contributed equally to this work.

Published online Jan. 22, 2015.

COPYRIGHT (C 2015 by the Society of Nuclear Medicine and Molecular Imaging, Inc. small papillary carcinomas, which is most likely due to the recent enhancement of diagnostic methods such as ultrasound and needle aspiration cytology. There is a large difference in the survival rates of patients with various thyroid carcinomas; patients with medullary carcinomas originating from the parafollicular $\mathrm{C}$ cells and rare, undifferentiated anaplastic carcinomas have worse survival rates than those with differentiated thyroid cancer (DTC). It has been well documented in the last $70 \mathrm{y}$ that DTC with the capacity to store iodine has a very good outcome compared with other cancers (1,3-5), with an average survival rate of more than $90 \%$. This fact is largely due to the possible administration of radioactive ${ }^{131} \mathrm{I}$ as a radioiodine therapy with the aim of complete remission, even in more advanced stages. Even high-risk patients have a favorable life expectancy, which has been improved through the implementation and analysis of high-quality clinical studies (6). Indeed, it has been shown that up to stage III, there is no difference between the survival of DTC patients and that of the general population. It remains controversial whether patients with stage IVA disease experience a shorter life-span because of their disease $(7,8)$. Nevertheless, there is no doubt that patients $45 \mathrm{y}$ old or older and diagnosed with distant metastases (stage IVC) have a worse survival rate than the general population. The presence of lateral lymph node metastases on recurrence of the disease after initial standardized treatment and the worse survival of such patients are still subject to debate $(7-11)$.

For staging of carcinomas, the TNM classification system of the American Joint Committee on Cancer (AJCC)-Union for International Cancer Control (UICC) is most commonly and widely accepted. The $\mathrm{T}$ categories are based on both tumor size and extrathyroid extension. In the current, seventh edition of the classification system, lymph node metastasis is classified into 2 categories: metastasis only in the central compartment is graded as $\mathrm{N} 1 \mathrm{a}$, and metastasis in the lateral or mediastinal compartment is graded as N1b (12). In a previous study of the life expectancy of DTC patients who underwent treatment at the Department of Nuclear Medicine, University Hospital of Münster, Münster, Germany, several interesting trends could be observed; however, they failed to reach statistical significance (7). Since then, we have been able to widen our database with a considerable number of patients and follow-up years. The aim of the present study was to reexamine the life expectancy of DTC patients to identify DTC patient subgroups with significantly altered life expectancy.

\section{MATERIALS AND METHODS}

A total of 2,476 patients presented between December 31, 1965, and April 23, 2013, at the Department of Nuclear Medicine, University Hospital of Münster, with diagnosed DTC. The University Hospital of 
Münster is a tertiary referral center serving approximately 5\%-10\% immigrants, which is also the average percentage of immigrants in the general population in Germany. Forty-eight patients had to be excluded, so that data from 2,428 patients were analyzed. A total of 1,823 patients had papillary thyroid carcinoma (PTC), 570 had follicular thyroid carcinoma (FTC), and the remaining 35 were diagnosed with poorly differentiated thyroid carcinoma (PDTC). Approximately threequarters of the patients were women $(26.2 \%$ were men). The median age at the time of diagnosis was $47 \mathrm{y}$, with a median follow-up time of $6.6 \mathrm{y}$.

TNM categorization was done according to the seventh edition of the AJCC-UICC TNM classification system after successful ablation of thyroid remnants with radioiodine, if applicable. Both posttherapeutic and initial diagnostic radioiodine scintigraphy results (6-9 mo after radioiodine remnant ablation) in combination with surgery and histology reports as well as other existing imaging (e.g., MR imaging, CT, ultrasound, and PET/CT) were used for the classification.

Treatment was administered according to current international standards (13-16). Follow-up data were obtained from patients' records at the Department of Nuclear Medicine, University Hospital of Münster, and from patients' family physicians. Overall survival analysis was performed by computing the time at risk from the date of diagnosis of thyroid cancer to the date of death or the date of last contact, whichever occurred first (observed overall survival). Observed overall survival rates were compared with expected survival rates. Expected survival was computed from data for the general population of former West Germany, excluding (West) Berlin, as published by the Federal Statistical Office (Wiesbaden, Germany) and controlling for sex, age, and calendar year periods. The Federal Statistical Office did not provide information on the expected survival of people more than $90 \mathrm{y}$ old. Therefore, DTC patients more than 90 y old were excluded from follow-up. Accordingly, the deaths that occurred after the age of $90 \mathrm{y}$ $(n=13)$ were excluded from the statistical comparison of expected and observed overall survival rates. One patient was omitted because she was more than $90 \mathrm{y}$ old at diagnosis. Other reasons for exclusion from the analysis were no specific death cause $(n=7)$, lack of an analytic histologic report for exact classification $(n=9)$, lack of information on sex $(n=1)$, and no follow-up $(n=30)$.

Matching of life expectancy for the control group was made individually for every year in the database up to 2008 . The calculation for patients from 2008 to 2013 was made from 2008 life expectancy tables. Histologic classification was taken from the corresponding pathologic report. For patients with 2 or more different entities of DTC, classification was based on the DTC with the worse outcome (PDTC $<$ FTC $<$ PTC). Similarly, in the presence of multifocal disease, the lesion with the largest diameter was chosen.

The institutional review boards of the Medical Association of Westphalia-Lippe and the Faculty of Medicine, University of Münster, approved this retrospective study, and the requirement to obtain informed consent was waived.

\section{Surgical Treatment and Radioiodine Therapy}

Most of the patients underwent total $(90.4 \%)$ or subtotal $(8.0 \%)$ thyroidectomy. Only a few received more limited surgery (hemithyroidectomy [1.5\%] or microdissection $[<0.1 \%]$ ). Neck dissection was applied in $39.9 \%$ of the cases. The histopathologic examination revealed that the residual status after the initial surgery was microscopic residual for $5.3 \%$ of the cases and macroscopic residual for $1.4 \%$ of the cases. Most of the patients $(91.1 \%)$ received a radioiodine remnant ablation with a median cumulative activity of approximately $4 \mathrm{GBq}$ of ${ }^{131} \mathrm{I}$ and a median tumor diameter of $1.5 \mathrm{~cm}$. Levothyroxine substitution/suppression therapy was administered according to international guidelines $(13,14)$.

A total of 234 patients had distant metastases (a total of 270 sites). Recurrences $(n=27)$ occurred in 21 patients. More detailed information is shown in Table 1. Follow-up included ultrasonography, measurement of
TABLE 1

Cohort Characteristics

\begin{tabular}{|c|c|}
\hline Characteristic & No. of patients \\
\hline \multicolumn{2}{|l|}{ Sex } \\
\hline Men & 637 \\
\hline Women & 1,791 \\
\hline \multicolumn{2}{|l|}{ Age at diagnosis } \\
\hline$<45$ y & 1,059 \\
\hline$\geq 45$ & 1,369 \\
\hline \multicolumn{2}{|l|}{ Histology } \\
\hline PTC & 1,823 \\
\hline FTC & 570 \\
\hline PDTC & 35 \\
\hline \multicolumn{2}{|l|}{$\begin{array}{l}\text { AJCC-UICC (seventh edition) } \\
\text { classification }\end{array}$} \\
\hline 1 & 1,577 \\
\hline II & 306 \\
\hline III & 300 \\
\hline IVA & 95 \\
\hline IVC & 150 \\
\hline \multicolumn{2}{|l|}{ Surgery } \\
\hline Thyroidectomy & 2,196 \\
\hline Subtotal thyroidectomy & 194 \\
\hline Hemithyroidectomy & 36 \\
\hline Microdissection & 2 \\
\hline Neck dissection & 969 \\
\hline \multicolumn{2}{|l|}{ 131| therapy } \\
\hline All & 2,213 \\
\hline PTC & 1,613 \\
\hline FTC & 566 \\
\hline PDTC & 34 \\
\hline \multicolumn{2}{|l|}{ Residual status after surgery } \\
\hline Ro & 2,265 \\
\hline $\mathrm{R} 1$ & 128 \\
\hline $\mathrm{R} 2$ & 35 \\
\hline Distant metastases & 234 (270 sites) \\
\hline Lung & 162 \\
\hline Bones & 68 \\
\hline Brain & 19 \\
\hline Mediastinal lymph nodes & 14 \\
\hline Liver & 6 \\
\hline Kidney & 1 \\
\hline Recurrence & 21 (27 sites) \\
\hline Thyroid & 9 \\
\hline Local lymph nodes & 13 \\
\hline Distant metastases & 5 \\
\hline
\end{tabular}

$\mathrm{R} 0=$ no residual; $\mathrm{R} 1=$ microscopic residual; $\mathrm{R} 2=$ macroscopic residual. 
the tumor marker thyroglobulin under thyroid-stimulating hormone suppression/stimulation, and diagnostic neck and whole-body scans with radioiodine according to international standards. Complex cases involved interdisciplinary discussions. Decisions on treatment were then often based on supplementary examinations, such as ${ }^{18} \mathrm{~F}$-FDG PET (or PET/CT) or needle aspiration biopsy. If locoregional recurrence was encountered, then decisions were made individually, with major consideration of compartment-oriented lymphadenectomy as the primary therapeutic option (17) as well as a second application of radioiodine therapy for residual lesions with the capacity to store iodine.

\section{Statistics}

Survival analysis methods were applied to compare expected and observed overall survival rates, that is, Kaplan-Meier estimation and the 1-sample log-rank test, conditional on patients' observed follow-up (18). Overall survival was calculated from the time of diagnosis until death or last contact. Event-free survival was calculated from the time of diagnosis until relapse, death, or last contact. The observed survival of DTC patients was compared with the expected survival of a matched general population as proposed by Finkelstein et al. (19). Matching criteria were age at diagnosis, sex, and calendar year. For each DTC patient, the individual mortality rate of a matched person in the general population was derived in each follow-up year after inclusion. The expected survival distribution and the expected number of deaths were obtained on the basis of individual mortality rates. The null hypothesis that mortality rates in the patient cohort were not different from the expected mortality rates was tested with the 1-sample logrank test $(20)$. We also calculated the standardized mortality ratio (SMR), defined as the ratio of the number of observed deaths in the patient cohort to the number of expected deaths in the matched general population over the analyzed time span. We calculated the $95 \%$ confidence interval (CI) of the SMR by using the method introduced by Finkelstein et al. (19).

In addition to the comparison with the reference population, subgroups were compared to identify potential risk factors for survival. Kaplan-Meier estimates and 2-sided log-rank tests were used to test for differences in survival. Cox proportional hazards models were used to estimate respective hazard ratios and $95 \%$ CIs. Inferential statistics were exploratory (hypothesis-generating), not confirmatory, and were interpreted accordingly. $P$ values of less than or equal to 0.05 were considered statistically significant. No adjustment for multiple testing was performed. Statistical analyses were performed with TIBCO Spotfire S+ (version 8.1 for Windows; TIBCO Software Inc.) and IBM SPSS Statistics (version 22.0 for Windows; released 2013; IBM Corp.).

\section{RESULTS}

The completeness index, defined as the quotient of the observed number of personyears divided by the expected number of person-years, for the entire collective at the last date of follow-up (April 23, 2013) was 0.8290 . Furthermore, the indices did not differ among the different stages (stage I: 0.8323; II: 0.8442; III: 0.7992; IVA: 0.7612; IVC: 0.8508).

Patients with stage I disease $(n=1,577)$ had a significantly better outcome than the general population (SMR: 0.61). There was no significant difference in survival between the control group and patients with stage II disease $(n=306)$ or stage III disease $(n=300)$. Similarly, there was no significant difference in survival between patients with stage IVA disease (T1$3 \mathrm{~N} 1 \mathrm{bM0} 0$ or T4aN0-1M0; $\geq 45$ y old; $n=95$ ) and the control group. However, patients with stage IVC disease (M1; $\geq 45$ y old; $n=150$ ) clearly had a worse survival rate (SMR: 2.45) (Fig. 1). SMRs, 95\% CIs, and $P$ values for various follow-up periods are shown in Table 2. It is noteworthy that there were no patients with stage IVB disease in our cohort, because this stage involves a large extrathyroid
FIGURE 1. Comparison of overall survival rates (according to seventh edition of AJCC-UICC TNM classification system) and $95 \%$ Cls for population of patients treated for DTC at University Hospital of Münster with survival rates for matched population. 
TABLE 2

Observed Versus Expected Numbers of Deaths in Each TNM Stage for Various Follow-up Periods

\begin{tabular}{|c|c|c|c|c|c|c|}
\hline \multirow[b]{2}{*}{ Follow-up } & \multirow[b]{2}{*}{ Parameter } & \multicolumn{5}{|c|}{ Value for stage*: } \\
\hline & & I $(1,577)$ & II (306) & III (300) & IVA (95) & IVC (150) \\
\hline \multirow[t]{4}{*}{$5 y$} & $\mathrm{O}$ & 13 & 11 & 19 & 6 & 38 \\
\hline & $E$ & 27.0 & 11.5 & 13.7 & 5.4 & 14.0 \\
\hline & SMR for O/E (95\% Cl) & $0.48(0.26-0.89)$ & $0.96(0.49-1.86)$ & 1.39 (0.84-2.31) & $1.11(0.46-2.69)$ & $2.72(1.90-3.91)$ \\
\hline & $P$ & 0.007 & 0.887 & 0.149 & 0.801 & $<0.001$ \\
\hline \multirow[t]{4}{*}{$10 y$} & 0 & 34 & 24 & 24 & 10 & 56 \\
\hline & $E$ & 51.3 & 22.7 & 24.7 & 9.7 & 22.0 \\
\hline & SMR for O/E (95\% Cl) & $0.66(0.45-0.97)$ & $1.06(0.67-1.66)$ & $0.97(0.62-1.53)$ & $1.03(0.51-2.06)$ & $2.54(1.89-3.42)$ \\
\hline & $P$ & 0.016 & 0.786 & 0.885 & 0.924 & $<0.001$ \\
\hline \multirow[t]{4}{*}{$15 y$} & $\mathrm{O}$ & 45 & 32 & 29 & 12 & 69 \\
\hline & $E$ & 69.6 & 31.6 & 31.9 & 11.2 & 26.6 \\
\hline & SMR for O/E (95\% Cl) & $0.65(0.46-0.90)$ & $1.01(0.68-1.50)$ & $0.91(0.60-1.38)$ & 1.07 (0.57-2.03) & $2.59(1.98-3.39)$ \\
\hline & $P$ & 0.003 & 0.936 & 0.613 & 0.810 & $<0.001$ \\
\hline \multirow[t]{4}{*}{$20 y$} & $\mathrm{O}$ & 51 & 37 & 32 & 14 & 73 \\
\hline & $E$ & 79.9 & 36.9 & 35.4 & 12.0 & 29.1 \\
\hline & SMR for O/E (95\% Cl) & $0.64(0.47-0.87)$ & $1.0(0.7-1.45)$ & $0.9(0.61-1.34)$ & $1.16(0.65-2.10)$ & $2.51(1.93-3.26)$ \\
\hline & $P$ & 0.001 & 0.983 & 0.571 & 0.569 & $<0.001$ \\
\hline \multirow[t]{4}{*}{ Maximum } & 0 & 52 & 38 & 33 & 14 & 75 \\
\hline & $E$ & 84.7 & 40.3 & 35.8 & 12.1 & 30.7 \\
\hline & SMR for O/E (95\% Cl) & $0.61(0.45-0.84)$ & $0.94(0.66-1.35)$ & $0.92(0.63-1.36)$ & $1.16(0.64-2.10)$ & $2.45(1.89-3.17)$ \\
\hline & $P$ & $<0.001$ & 0.713 & 0.640 & 0.576 & $<0.001$ \\
\hline
\end{tabular}

growth, which is rare in countries with a highly developed medical system (such as Germany).

Given that there is a considerable difference in biologic behavior of tumors among patients of different ages and given the large number of patients in the cohort that we studied, the influence of age was analyzed further by subdividing the cohort into different age categories, as follows: juvenile (0-19 y old), early adulthood (20-44 y old), middle adulthood (45-59 y old), and late adulthood (60 y old or older) (Supplemental Figs. 1-6 and Supplemental Table 1) (supplemental materials are available at http://jnm. snmjournals.org). In summary, the most important finding was that patients who were $20-45$ y old and had distant metastases at diagnosis (stage II) had a significantly and considerably increased SMR. In contrast, patients who were more than $45 \mathrm{y}$ old and had stage II disease had a normal mortality rate. Furthermore, as we already found previously (7), the mortality rate was significantly increased in patients who had stage IVC disease and were 45-60 y old and more than $60 \mathrm{y}$ old. On the other hand, a significantly lower mortality rate was observed in elderly patients $(\geq 60 \mathrm{y}$ old) who had stage I disease, that is, patients with small primary tumors $(\leq 2 \mathrm{~cm})$ but no lymph node or distant metastases.

Further statistical analysis of risk parameters for overall survival and event-free survival revealed differences $(P \leq 0.05)$ in sex, age, distant metastases, and histology. Women had a more favorable outcome than men, and the presence of metastases was the major predictive factor for survival. Furthermore, patients $45 \mathrm{y}$ old or older at the time of diagnosis had worse event-free survival than younger patients. With regard to histology, patients with PDTC had the worst outcome, and those with PTC had the most favorable outcome. The presence of macroscopic residual was indicative of a poorer outcome than the presence of no residual or microscopic residual. With regard to lymph node status, only the comparison between $\mathrm{N} 0$ and $\mathrm{N} 1 \mathrm{~b}$ revealed a significant difference in overall survival and event-free survival in favor of N0. Statistical analysis of SMRs, 95\% confidence intervals, and $P$ values for overall survival and event-free survival are shown in Table 3.

\section{DISCUSSION}

In recent years, the incidence of thyroid cancer has been increasing throughout the world. This increase is mainly due to earlier diagnosis through improved diagnostic procedures, including ultrasound and fine-needle aspiration cytology, as well as thin-section histopathologic examination of thyroid originally surgically removed for noncancerous disease. The enhanced incidence is reflected in a marked increase in the number of patients included in the Münster Thyroid Cancer Registry in recent years.

There are numerous studies on the treatment of DTC. Surprisingly, very few address the difference in survival between patients with DTC and the general population $(7,8,21)$. As Eustatia-Rutten et al. stated, only 2 of 28 reviewed articles on DTC outcomes had initial and continued standardized treatments through the 
TABLE 3

Statistical Analysis of Risk Parameters for Overall Survival and Event-Free Survival

\begin{tabular}{|c|c|c|}
\hline Parameter & Hazard ratio $(95 \% \mathrm{Cl})$ & $P$ \\
\hline \multirow[t]{2}{*}{ Women vs. men } & $0.512(0.391-0.672)$ & $<0.001$ \\
\hline & $0.488(0.376-0.633)$ & $<0.001$ \\
\hline \multirow[t]{2}{*}{ M0 vs. M1 } & $0.190(0.145-0.2499)$ & $<0.0001$ \\
\hline & $0.187(0.144-0.243)$ & $<0.0001$ \\
\hline \multirow[t]{2}{*}{$\geq 45$ y vs. $<45$ y } & $10.777(6.645-17.477)$ & $<0.0001$ \\
\hline & $8.056(5.316-12.208)$ & $<0.0001$ \\
\hline \multirow[t]{2}{*}{ FTC vs. PDTC } & $0.1462(0.086-0.248)$ & $<0.0001$ \\
\hline & $0.166(0.097-0.280)$ & $<0.0001$ \\
\hline \multirow[t]{2}{*}{ FTC vs. PTC } & $1.763(1.332-2.333)$ & $<0.0001$ \\
\hline & 1.825 (1.395-2.387) & $<0.0001$ \\
\hline \multirow[t]{2}{*}{ PDTC vs. PTC } & $12.060(7.227-20.125)$ & $<0.0001$ \\
\hline & 10.977 (6.597-18.265) & $<0.0001$ \\
\hline \multirow[t]{2}{*}{ R0 vs. R2 } & $0.316(0.172-0.582)$ & $<0.0002$ \\
\hline & $0.2685(0.153-0.471)$ & $<0.0001$ \\
\hline \multirow[t]{2}{*}{ R1 vs. R2 } & $0.420(0.193-0.916)$ & 0.0292 \\
\hline & $0.386(0.187-0.795)$ & 0.0098 \\
\hline \multirow[t]{2}{*}{ N0 vs. N1b } & $0.501(0.359-0.698)$ & $<0.0001$ \\
\hline & $0.486(0.353-0.668)$ & $<0.0001$ \\
\hline
\end{tabular}

$\mathrm{R} 0=$ no residual; $\mathrm{R} 1=$ microscopic residual; $\mathrm{R} 2=$ macroscopic residual.

follow-up (22). Furthermore, studies on the survival of patients with DTC have often failed to classify the tumors with the AJCC-UICC classification system, which appears to be the most powerful method for clinical treatment and risk stratification (23-25).

Updating the formerly published Münster Thyroid Cancer Registry (7) has created one of the largest cohorts of uniformly treated DTC patients known so far. Accordingly, the number of patients has increased from 1,497 patients in 2008 to 2,428 patients in 2013 , and the number of available follow-up years has increased by $78 \%$. In contrast to the findings of the previous study (7), a small but significant improvement in survival (increase in the SMR from 0.77 to 0.61 ) has been documented for patients with stage I thyroid cancer relative to the general population. This finding may be due in part to selection bias because people who undergo regular preventive medical checkups may have the opportunity for earlier thyroid cancer diagnosis. Furthermore, the increased follow-up time-in particular, due to the addition of patients diagnosed since 1966-may accentuate the benefits of the lifelong medical surveillance of patients included in the clinical study. Thus, a healthier lifestyle is promoted in these patients through continuous monitoring and patients' reflection on health parameters, such as body weight, physical training, blood pressure, and heart rate. In addition, the initiation of earlier medical clarification of other diseases leads to a long-term prognostic advantage in these patients. Another fact supporting the hypothesis is that a significantly better outcome was shown only for the elderly patients with stage I disease. This group, being at higher risk for health hazards, will benefit the most from intense medical surveillance within the first few years of the initial diagnosis.
Consequently, other diseases can be revealed at an early stage, contributing to an improvement in survival. Furthermore, the introduction of dedicated SPECT/CT and PET/CT systems may facilitate the timely detection of tumor recurrence and metastasis. It is conceivable that deaths in stage I were missed more often than deaths in other stages because the good prognosis would have led to the patients no longer being monitored after a long recurrencefree period, especially when more serious diseases may have made it difficult to attend regular clinic visits. However, this possibility is highly unlikely because the completeness index for these patients was as good as that for patients with higher stages of disease.

A "healthy patient phenomenon" was reported for a population in which the effect of pregnancy on overall survival after the diagnosis of early-stage breast cancer (26) was studied. The investigators observed a better outcome for patients who became pregnant after the diagnosis of early-stage breast cancer than for nonpregnant matched patients and interpreted the result as a "healthy mother" effect. The authors stated that this observation may merely represent a "healthy patient" bias, in that women with a smaller, subclinical tumor load and therefore a better prognosis may be more likely to attempt or to achieve a subsequent pregnancy. Another study reported better survival of patients undergoing in vitro fertilization than of the general population (27). These authors also suggested a "healthy patient effect" based on the notion that the least healthy women in a community may be deterred from planning a pregnancy or accessing in vitro fertilization services; that is, women who are planning a pregnancy may be healthier than other women of the same age.

One of the most frequently debated points in publications on the survival of DTC patients is the difference in outcome and recurrence between patients with lateral lymph node metastases and other patients. Although Bhattacharyya asserted that cervical lymph node metastasis does not influence survival in DTC patients (11), Podnos et al. reported the opposite result (10). Also, 2 of the most recent studies pertaining to this question in Germany had different results. Although Verburg et al. emphasized the poorer survival of patients with lateral lymph node metastases (8), Vrachimis et al. reported no reduction in the survival of patients with stage IVA disease (7). In the present study, we confirmed our previous results, showing no worsening of the outcome for patients with stage IVA disease. These results enhanced the opinion expressed by Verburg et al. (8), who suggested that their results might have been due to earlier compartment-oriented surgical intervention instead of watchful waiting and subsequent additional radioiodine therapy (28).

Although reporting a good outcome for patients with stage I-IVA disease, the present study and other studies $(7,8,21)$ showed that patients with stage IVC disease still had a clearly poorer outcome than the general population. Accordingly, especially at this stage, it is important to conduct further research on definitive individual treatments with modern, promising therapies, such as tyrosine kinase inhibitors, radioimmunotherapy and receptor therapy, use of bisphosphonates for metastases in the bones, or stereotactic radiation of brain metastases (29). Another important finding of the present study was that the conventionally held belief that young adults with distant metastases have a very good prognosis, likely with an unimpaired life expectancy, may not conform to reality, because the present study was the first to show (to our knowledge) a significantly increased SMR for young adults (20-45 y old) with distant metastases at the time of diagnosis. This 
finding is in contrast to that reported by Verburg et al. (8), who did not find a lower relative survival rate for this group of patients.

The present study did have some limitations. One of the most challenging aspects of the comparison of our cohort with the German general population was the formation of a valid cohort. We concede that, although this goal was achieved for Germany, the present study is not necessarily representative for other countries with different populations and geographic backgrounds. The reason is that the incidences of DTC differ greatly (30-32), with potential differences in genetics; these can influence prognosis and outcome (2). This potential bias is also represented by the fact that the present study did not include any patients with stage IVB disease, with a large extrathyroid growth, because it does not occur frequently in highly industrialized countries (such as Germany). Patients were excluded at the age of 90 until the year 2000 because the Federal Statistical Office did not provide any further information beyond this age. As a consequence, 13 patients had to be excluded; this exclusion could have led to minor biases represented in inaccurate descending of the survival curves. The median follow-up time of $6.6 \mathrm{y}$ was comparable to those in other, similar studies, and the surveillance period was very long. Nevertheless, because of the very good prognosis for most of the patients, the time for making statements pertaining to long-term recurrence rates in the patients is relatively short; this topic could be part of a future analysis. However, this limitation was partially compensated for by the adequate patient-years assessed in the present study.

\section{CONCLUSION}

Older patients ( $>60$ y old) with more limited disease (stage I) paradoxically had better survival rates than would be expected on the basis of age and sex, whereas young adults (20-45 y old) as well as any patient more than $45 \mathrm{y}$ old and with distant metastases had increased mortality. For all other DTC patients, regardless of age or TNM stage, no significant difference in survival was found.

\section{DISCLOSURE}

The costs of publication of this article were defrayed in part by the payment of page charges. Therefore, and solely to indicate this fact, this article is hereby marked "advertisement" in accordance with 18 USC section 1734 . No potential conflict of interest relevant to this article was reported.

\section{REFERENCES}

1. Schlumberger MJ. Papillary and follicular thyroid carcinoma. N Engl J Med. 1998;338:297-306.

2. Lupi C, Giannini R, Ugolini C, et al. Association of BRAF V600E mutation with poor clinicopathological outcome in 500 consecutive cases of papillary thyroid carcinoma. J Clin Endocrinol Metab. 2007;92:4085-4090.

3. Verburg FA, Mäder U, Luster M, Reiners C. Histology does not influence prognosis in differentiated thyroid carcinoma when accounting for age, tumor diameter, invasive growth and metastases. Eur J Endocrinol. 2009;160:619-624.

4. Riemann B, Schober O; MSDS Study Group. Are "high-risk" patients with differentiated thyroid cancer "healthy patients"? Nuklearmedizin. 2008;47:179180.

5. Volante M, Collini P, Nikiforov YE, et al. Poorly differentiated thyroid carcinoma: the Turin proposal for the use of uniform diagnostic criteria and an algorithmic diagnostic approach. Am J Surg Pathol. 2007;31:1256-1264.

6. Krämer JA, Schmid KW, Dralle H, et al. Primary tumor size is a prognostic parameter in patients suffering from differentiated thyroid carcinoma with ex- trathyroidal growth: results of the MSDS trial. Eur J Endocrinol. 2010;163:637644.

7. Vrachimis A, Riemann B, Gerss J, Maier T, Schober O. Peace of mind for patients with differentiated thyroid cancer? Nuklearmedizin. 2013;52:115-120.

8. Verburg FA, Mäder U, Tanase K, et al. Life expectancy is reduced in differentiated thyroid cancer patients $\geq 45$ years old with extensive local tumor invasion, lateral lymph node, or distant metastases at diagnosis and normal in all other DTC patients. J Clin Endocrinol Metab. 2013;98:172-180.

9. Reiners C, Hanscheid H, Luster M, et al. Radioiodine for remnant ablation and therapy of metastatic disease. Nat Rev Endocrinol. 2011;7:589-595.

10. Podnos YD, Smith D, Wagman LD, Ellenhorn JD. The implication of lymph node metastasis on survival in patients with well-differentiated thyroid cancer. Am Surg. 2005;71:731-734.

11. Bhattacharyya N. A population-based analysis of survival factors in differentiated and medullary thyroid carcinoma. Otolaryngol Head Neck Surg. 2003; 128:115-123.

12. Sobin LH, Compton CC. TNM seventh edition: what's new, what's changedcommunication from the International Union Against Cancer and the American Joint Committee on Cancer. Cancer. 2010;116:5336-5339.

13. Cooper DS, Doherty GM, Haugen BR, et al. Revised American Thyroid Association management guidelines for patients with thyroid nodules and differentiated thyroid cancer. Thyroid. 2009;19:1167-1214.

14. Pacini F, Schlumberger M, Dralle H, Elisei R, Smit JW, Wiersinga W. European consensus for the management of patients with differentiated thyroid carcinoma of the follicular epithelium. Eur J Endocrinol. 2006;154:787-803.

15. Luster M, Clarke SE, Dietlein M, et al. Guidelines for radioiodine therapy of differentiated thyroid cancer. Eur J Nucl Med Mol Imaging. 2008;35:1941-1959.

16. Dietlein M, Dressler J, Eschner W, et al. Procedure guidelines for radioiodine therapy of differentiated thyroid cancer (version 3). Nuklearmedizin. 2007; 46:213-219.

17. Lerch H, Schober O, Kuwert T, Saur HB. Survival of differentiated thyroid carcinoma studied in 500 patients. J Clin Oncol. 1997;15:2067-2075.

18. Kaplan EL, Meier P. Nonparametric estimation from incomplete observations. J Am Stat Assoc. 1958;53:457-481.

19. Finkelstein DM, Muzikansky A, Schoenfeld D. Comparing survival of a sample to that of a standard population. J Natl Cancer Inst. 2003;95:1434-1439.

20. Woolson RF. Rank tests and a one-sample Logrank test for comparing observed survival-data to a standard population. Biometrics. 1981;37:687-696.

21. Links TP, van Tol KM, Jager PL, et al. Life expectancy in differentiated thyroid cancer: a novel approach to survival analysis. Endocr Relat Cancer. 2005; 12:273-280.

22. Eustatia-Rutten CF, Corssmit EP, Biermasz NR, et al. Survival and death causes in differentiated thyroid carcinoma. J Clin Endocrinol Metab. 2006;91:313-319.

23. Brierley JD, Panzarella T, Tsang RW, Gospodarowicz MK, O'Sullivan B. A comparison of different staging systems predictability of patient outcome: thyroid carcinoma as an example. Cancer. 1997;79:2414-2423.

24. Passler C, Prager G, Scheuba C, Kaserer K, Zettinig G, Niederle B. Application of staging systems for differentiated thyroid carcinoma in an endemic goiter region with iodine substitution. Ann Surg. 2003;237:227-234.

25. Verburg FA, Mäder U, Kruitwagen CL, Luster M, Reiners C. A comparison of prognostic classification systems for differentiated thyroid carcinoma. Clin Endocrinol (Oxf). 2010;72:830-838.

26. Gelber S, Coates AS, Goldhirsch A, et al. International Breast Cancer Study Group: effect of pregnancy on overall survival after the diagnosis of early-stage breast cancer. J Clin Oncol. 2001;19:1671-1675.

27. Venn A, Hemminki E, Watson L, Bruinsma F, Healy D. Mortality in a cohort of IVF patients. Hum Reprod. 2001;16:2691-2696.

28. Verburg F, Weber T, Luster M. ${ }^{131} \mathrm{I}$ sodium-iodide versus ${ }^{56} \mathrm{Fe}$ surgical steel: which is better for lateral lymph node metastases in differentiated thyroid cancer patients? Nuklearmedizin. 2013;52:113-114.

29. Vrachimis A, Schmid KW, Jürgens H, Schober O, Weckesser M, Riemann B. Cerebral metastases from thyroid carcinoma: complete remission following radioiodine treatment. Dtsch Arztebl Int. 2013;110:861-866.

30. Kolonel LN, Hankin JH, Wilkens LR, Fukunaga FH, Hinds MW. An epidemiologic study on thyroid cancer in Hawaii. Cancer Causes Control. 1990;1:223234.

31. Mulla ZD, Margo CE. Primary malignancies of the thyroid: epidemiologic analysis of the Florida Cancer Data System registry. Ann Epidemiol. 2000;10:24-30.

32. Pettersson B, Coleman MP, Ron E, Adami HO. Iodine supplementation in Sweden and regional trends in thyroid cancer incidence by histopathologic type. Int $J$ Cancer. 1996;65:13-19. 